\title{
Dr. Mark Wainberg on old battles and new frontiers in HIV
}

$\mathrm{F}$ ew Canadian researchers were interested in HIV when Dr. Mark Wainberg started working in the field in the 1980s.

"In the early days of the epidemic, there were a lot of naysayers and people who thought this would never be important."

Wainberg's achievements in HIV research, including the 1989 identification of lamivudine, or 3TC, one of the most widely used HIV treatments, have since earned him a place among Canada's medical luminaries.

In April, Wainberg will join the current 113 laureates in the Canadian Medical Hall of Fame.

A passionate advocate for access to antiretroviral drugs, Wainberg cites his tenure as president of the International AIDS Society from 1998 to 2000 as the highlight of his career. While president, he brought unprecedented attention to gaps in access to HIV treatment in the developing world by bringing the International Conference on AIDS in 2000 to Durban, South Africa.

The disparities he highlighted in the early 2000s continue today. A recent report by Médecins Sans Frontières warned that antiretroviral medicines are still not making their way to patients in sub-Saharan Africa, even when sufficient stocks are present. For example, nationwide surveys conducted in South Africa showed that up to $25 \%$ of local health centres were unable to dispense the complete amount of one or more HIV or tuberculosis medications.

As such, Wainberg considers his life's work unfinished and has recently turned his attention to achieving a cure for the virus.

Wainberg reflected on his legacy and current work in a CMAJ podcast interview. Excerpts and podcast have been edited for length.

Tune in to the podcast via Sound Cloud.com

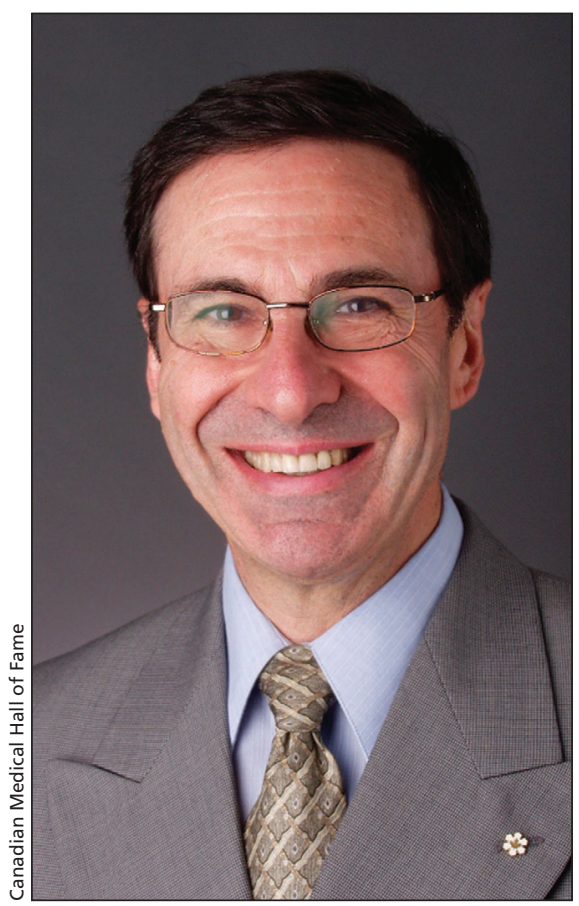

The Canadian Medical Hall of Fame will soon induct renowned HIV researcher Dr. Mark Wainberg.

How did you first become involved in AIDS research?

I had done a sabbatical in the early 80 s at the National Institutes of Health [in Washington, DC] in the laboratory of Dr. Robert Gallo, who is widely credited with being one of the codiscoverers of HIV. Dr. Gallo sent me all of the reagents that I needed to become the first scientist in Canada who was equipped to do lab work on HIV.

What were the biggest challenges you faced upon becoming president of the International AIDS Society in 1998?

It was obvious that we had turned a corner of sorts. We now had many more drugs that were working better. What was also obvious is that these drugs were available primarily to rich developed countries, and that most people in developing countries were not able to have any kind of HIV drug access. So we made a decision as the International AIDS Society that one way of highlighting this disparity was to bring our conference to South Africa, which then and now had the highest number of HIV infections in the world.

What was the impact of the conference?

Politicians everywhere started to take note of this disparity. Do you know how many people on the African continent had access to antiretroviral therapy in 2000? The figure was 7000 people out of at least 10 or 11 million HIV-infected individuals. Today, the numbers of people who have drug access throughout Africa is at least 8 to 10 million. It's hard for me to compare anything I've done in my life with that.

Looking forward, what do you see as promising avenues for HIV prevention?

There's a new paper coming out in the New England Journal of Medicine that is going to show that — let's say I'm a gay man and I know that Friday night is my big night - if I just take [Truvada, a pre-exposure prophylaxis combination drug] on Friday morning and Saturday morning, I'm going to be protected. And that's pretty good, because if you only take your drug twice a week instead of seven days a week, we will save costs and we will cut down on side effects.

\section{You're also working towards a cure} for HIV?

Our own work now is focused on one of the newer drugs called dolutegravir. It's an integrase inhibitor and, remarkably, no one has ever developed resistance against this compound. So we developed the notion that you cannot get resistance against this drug because the mutations that would confer resistance against it and that are selected by the drug simultaneously compromise the ability of the virus to grow. If you have a drug that you can't get resistance against, we can stop 
treatment and then put somebody back on treatment later, and maybe this will give us a way to flush out the cells that are latently infected.

What advice would you give young researchers entering your field today?
Young people need to know HIV is still a very vital and important problem, but it's not the only one. And scientists have to be able to switch gears pretty fast if they want to remain competitive. I can tell you it's pretty hard to get a grant to work on the hepatitis $\mathrm{C}$ virus in relative terms since the curative strate- gies have been developed. We haven't figured out yet how to cure HIV, but hopefully that is going to happen, and if and when it does, then HIV scientists have to be nimble enough to switch fields. - Lauren Vogel, CMAJ

CMAJ 2016. DOI:10.1503/cmaj.109-5204 